\title{
Physical Activity and Bone Health in Men: A Systematic Review and Meta-Analysis
}

\author{
Maureen C. Ashe ${ }^{1,2}$, Isis Kelly dos Santos ${ }^{3}$, Nicola Y. Edwards ${ }^{1}$, Laura A. Burnett ${ }^{1}$, Rosanne Barnes ${ }^{1}$, Lena Fleig ${ }^{4}$, \\ Joseph H. Puyat ${ }^{5}$, Joanna E. M. Sale ${ }^{6}$, Heather A. McKay ${ }^{1,2}$, Lora M Giangregorio ${ }^{7,8}$ \\ ${ }^{1}$ Centre for Hip Health and Mobility, The University of British Columbia, Vancouver; \\ ${ }^{2}$ Department of Family Practice, The University of British Columbia, Vancouver, Canada \\ ${ }^{3}$ Department of Health Sciences, Federal University of Rio Grande do Norte, Natal, Brazil \\ ${ }^{4}$ Department of Psychology, MSB Medical School Berlin, Berlin, Germany \\ ${ }^{5}$ School of Population and Public Health, The University of British Columbia, Vancouver; \\ ${ }^{6}$ Musculoskeletal Health and Outcomes Research, Li Ka Shing Knowledge Institute, St. Michael's Hospital, Unity Health, Toronto; \\ ${ }^{7}$ Department of Kinesiology and Schlegel Research Institute for Aging, University of Waterloo, Waterloo; \\ ${ }^{8}$ Schlegel-University of Waterloo Research Institute for Aging, Waterloo, Canada
}

Corresponding author

Maureen C. Ashe

Department of Family Practice, The University of British Columbia, 3rd Floor, 5950 University

Boulevard, Vancouver BC V6T 1Z3, Canada

Tel: +1-604-675-2574

Fax: +1-604-675-2576

E-mail: maureen.ashe@ubc.ca

Received: September 17, 2020

Revised: December 30, 2020

Accepted: December 31, 2020
Background: Research on osteoporosis and physical activity often focuses on women. We aimed to conduct a systematic review to assess the benefits and harms of physical activity interventions for men's bone health. Methods: We used standard methods and searched for randomized controlled trials (RCTs) (duration, $\geq 6$ months) published in all languages across multiple databases and trial registries. The last search was conducted on July 22, 2020. Results: We included 11 studies (14 publications), resulting in a sample of $\mathrm{N}=723$ men (range, 17-132 participants). We found low-certainty evidence that physical activity has little influence on the areal bone mineral density (aBMD) at the total hip (5 RCTs, N=324; mean difference [MD], 0.03 [95 confidence interval (Cl), 0.01 to 0.05 ]) and little or no influence on the aBMD at the femoral neck ( $3 \mathrm{RCTs}, \mathrm{N}=186 ; \mathrm{MD}, 0.00$ [ $95 \% \mathrm{Cl},-0.04$ to 0.04$]$ ), lumbar spine (3 RCTs; $\mathrm{N}=213 ; \mathrm{MD}, 0.05$ [95\% $\mathrm{Cl},-0.01$ to 0.11$]$ ), and whole body (4 RCTs, $\mathrm{N}=203 ; \mathrm{MD},-0.00$ [95\% Cl, -0.03 to 0.02$]$ ). Conclusions: We found low-certainty evidence that physical activity ( $\geq 6$ months) has some effect on the total hip in men, but new evidence may change this finding. This review highlights the gap in the evidence on specific intervention prescriptions that can benefit the bone geometry, structure, microarchitecture, and, ultimately, bone strength in men. Future research should engage in comprehensive reporting of harms, quality of life outcomes, advanced imaging findings, and long-term interventions.

Key Words: Bone and bones $\cdot$ Exercise $\cdot$ Male $\cdot$ Musculoskeletal diseases

\section{INTRODUCTION}

Copyright (C) 2021 The Korean Society for Bone and Mineral Research

This is an Open Access article distributed under the terms of the Creative Commons Attribution Non-Commercial License (https://creativecommons.org/licenses/by-nc/4.0/) which permits unrestricted non-commercial use, distribution, and reproduction in any medium, provided the original work is properly cited.

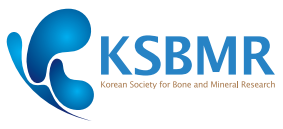

Postmenopausal women have a higher risk of low-trauma fractures, but up to $25 \%$ of men have an osteoporosis-related fracture in their lifetime, $[1,2]$ and they may have worse outcomes compared with women after hip fractures.[3] Older men are more likely to have a low-trauma fracture than a diagnosis of prostate or lung cancer,[4] but men are frequently not considered to have osteoporosis and or osteoporosis-related low-trauma fractures. As few as $5 \%$ of men with a lowtrauma fracture are investigated for their bone health.[5] The economic burden is 
substantial: In Canada in 2007-2008, the annual cost of osteoporotic fractures in men aged 50 years and older was estimated at CAD \$570 M.[6]

Physical activity is an important prevention strategy across the lifespan to maximize bone health in the later years. It can provide health benefits across body systems, including enhanced bone health [7] and reduced risk of falls.[8] In the aging skeleton most of our knowledge about the role of physical activity for maintaining bone mass/density/ strength is from studies of postmenopausal women. For this review, we focused on men and aimed to synthesize the evidence on the effect of physical activity interventions on fracture (when available) quality of life (QOL), and bone strength and its related outcomes (density). Our objective was to assess the benefits and harms of physical activity interventions for preventing or managing osteoporosis in men aged 19 years and older. This current review updates previous work exploring the effect of physical activity and bone health in men.[9-13]

\section{METHODS}

We followed the recommendations outlined in Preferred Reporting Items for Systematic Reviews and Meta-Analyses (PRISMA) [14] and Grading of Recommendations Assessment, Development, and Evaluation (GRADE).[15]

\section{Types of study designs}

We included randomized controlled trials (RCTs), cluster RCTs and cross-over trials that examined the effects of a physical activity intervention ( $\geq 6$ month duration) on bone outcomes in men. We included interventions that had a full-text report available for review, and excluded abstractonly citations, theses, and grey literature.

\section{Types of participants}

We included trials that enrolled community-dwelling men (aged 19 years and older) with or without a diagnosis of osteoporosis. We also had interventions with both men and women where the analysis of major outcomes (e.g., bone health) was reported separately.

\section{Types of interventions}

We included trials that compared physical activity interventions with usual care, no intervention, and/or wait list control. Interventions included aerobic, high impact loading and resistance training, sport, Tai Chi, and combined programs. We excluded interventions designed specifically for weight loss, interventions that contained supplements, or included clinical populations (other than studies with men with low bone mass).

\section{Types of outcomes}

We included the following major outcomes: Number and rate of (1) vertebral; (2) non-vertebral hip; and (3) other fractures; (4) QOL; and (5) adverse events (withdrawals, serious and total). If fracture data were unavailable, we substituted the first 3 outcomes with total hip areal bone mineral density ( $a B M D)$, femoral neck aBMD, lumbar spine aBMD, and whole-body aBMD. We planned to include volumetric outcomes (density and strength), if available. We also had attendance as a minor outcome.

We identified items from the following databases: CINAHL via EBSCO (1980-present); The Cochrane Library via Wiley including the Cochrane Central Register of Controlled Trials (CENTRAL), the Cochrane Database of Systematic Reviews, the Database of Reviews of Effects (DARE), and the Health Technology Assessment database (HTA); EMBASE via Ovid (1947-present); Google Scholar (Advanced Feature: keywords in title); MEDLINE via Ovid (1946 to present); and SPORTDiscus. The electronic search strategy for MEDLINE is provided in Supplementary Table 1. We adapted this search strategy for use with other databases. We used the sensitivity and precision-maximising version filter designed to identify clinical trials.[16] To limit the search to 'men' we used a filter validated for this purpose.[17] Two authors (blinded for peer-review) reviewed the reference lists of relevant systematic reviews. We searched for errata or retractions of included studies published in full text on PubMed and reported the date this was done within the review. We searched the World Health Organization International Clinical Trials Registry Platform and ClinicalTrials. gov to identify any interventions in progress. We did not restrict the search by language or publication status. The last search was conducted on July 22, 2020.

Following development of the search strategy, one author (blinded for peer-review) conducted the database searches and uploaded them to Covidence systematic review software (Covidence; Veritas Health Innovation, Melbourne, Australia; www.covidence.org), where duplicates were re- 
moved. Next, 2 authors (blinded for peer-review) independently screened titles and abstracts (Level 1). We retrieved the full-text records (Level 2) and 2 review authors (blinded for peer-review) independently screened the full text and identified records for inclusion, and recorded reasons for exclusion of the ineligible records. We resolved any disagreement through discussion with a third author (blinded for peer-review). We collated multiple reports of the same intervention so that each study was the unit of interest in the review. We recorded the selection process in sufficient detail to complete a PRISMA flow diagram using data from Covidence.

We used a data collection form for intervention characteristics and outcome data and piloted it on 2 interventions in the review. One review author (blinded for peer-review) extracted intervention characteristics from included interventions, and 5 review authors (blinded for peer-review) confirmed the extracted information. Three authors (blinded for peer-review) checked all data for accuracy. Another author (blinded for peer-review) was available to confirm data extraction and or adjudicate discrepancies. Data were obtained from published literature. For included studies, we obtained additional information or clarification of data through email correspondence with authors.

Two of 4 review authors (blinded for peer-review) independently assessed the risk of bias for each intervention using the criteria outlined by the Cochrane Collaboration. [18] We resolved any disagreements by discussion or by involving another author (blinded for peer-review). To assess outcome reporting bias, we checked trial registrations against published reports.

We analysed continuous data as mean difference with 95\% confidence intervals ( $\mathrm{Cls}$ ) between groups, and reported either final values or change data for each outcome (depending on availability). We calculated the mean difference based on the number of men analysed at the final time point. If the number of men analysed was not presented for each time point, we used the number of randomized men in each group at baseline. We contacted authors to verify key intervention characteristics and obtain missing numerical outcome data where possible (e.g., when data were not available for all participants).

Four authors (blinded for peer-review) independently assessed the evidence and 4 authors (blinded for peer-review) confirmed these findings. We used the 5 GRADE con- siderations (intervention limitations, consistency of effect, imprecision, indirectness, and publication bias) to assess the body of evidence that contributed data to the metaanalysis for the pre-specified outcomes. We used methods and recommendations from the Cochrane Collaboration using the GRADEpro Guideline Development Tool Software (McMaster University, 2015).[19] We justified all decisions to down- or up-grade the quality of interventions. Two authors (blinded for peer-review) confirmed these findings at the conclusion of the review process.

We undertook meta-analysis only where this was meaningful (i.e., if the treatments, participants, and the underlying clinical question were similar enough for pooling to make sense). We reported results using a fixed-effects model, or random-effects model when there was (clinical and statistical) heterogeneity between study participants and physical activity interventions. For interventions with 2 or more arms, in the meta-analysis we combined the 2 interventions using standard procedures, as outlined in Section 9.3.9 of the Cochrane Handbook.[20] The effect estimates were computed using the DerSimonian-Laird method with the Knapp-Hartung small-sample adjustments.[21] We used R (version 4.0.3; The R Foundation for Statistical Computing, Vienna, Austria) [22] using the meta-package.[23]

We planned to carry out subgroup analysis of outcomes by 2 age groups (men aged 19-44 years and men aged $45+$ years); and sensitivity analysis for outcomes from interventions with low risk of bias versus high risk of bias for (1) allocation concealment; and (2) blinding of outcome assessor. We conducted additional sensitivity analysis for the type of statistical modeling (random vs. fixed effects) and men with and without a diagnosis of osteoporosis.

\section{RESULTS}

Table 1 is a summary of the main differences between our review protocol and full systematic review. See Figure 1 for the PRISMA flow diagram.[14] We identified 2,919 unique records from bibliographic database searches, 1 record from searching related systematic review publications, and one record from a trial we identified on a registry. There were 324 duplicates removed, leaving 2,597 records reviewed at Level 1. We screened 114 studies at Level 2 and included 14 publications and 11 studies in the review. We identified 2 instances of records reporting on the same 
Table 1. Major differences between the original protocol and full systematic review

\begin{tabular}{|c|c|}
\hline 1 & Instead of 2 authors (as stated in the protocol), 1 librarian developed the strategy and 1 author ran the search. \\
\hline 2 & $\begin{array}{l}\text { Contrary to the protocol, we did not look at presence of chronic disease and disease duration, we added the exclusion criteria to exclude } \\
\text { interventions that enrolled clinical populations (except osteoporosis). Thus, the subgroup analysis comparing interventions of men with } \\
\text { chronic disease and healthy men no longer applied to the review. }\end{array}$ \\
\hline 3 & $\begin{array}{l}\text { We did not include studies focused on comparing } 2 \text { physical activity interventions or intensities, and do not provide a description or } \\
\text { discussion of dose-response in the manuscript. }\end{array}$ \\
\hline 4 & $\begin{array}{l}\text { In the data extraction and management section we changed the protocol to: "1 review author (blinded for peer-review) extracted intervention } \\
\text { characteristics from included interventions and } 5 \text { review authors (blinded for peer-review) confirmed the extracted information. Finally, } 3 \\
\text { authors (blinded for peer-review) checked all data for accuracy." }\end{array}$ \\
\hline 5 & We looked at program compliance and adverse events separately. \\
\hline 6 & In the risk of bias section, we determined: greater than $20 \%$ loss of data was consider high risk. \\
\hline 7 & $\begin{array}{l}\text { We conducted a sensitivity analysis for type of statistical modelling (random vs. fixed effects) and for men with and without osteoporosis } \\
\text { diagnosis. }\end{array}$ \\
\hline
\end{tabular}

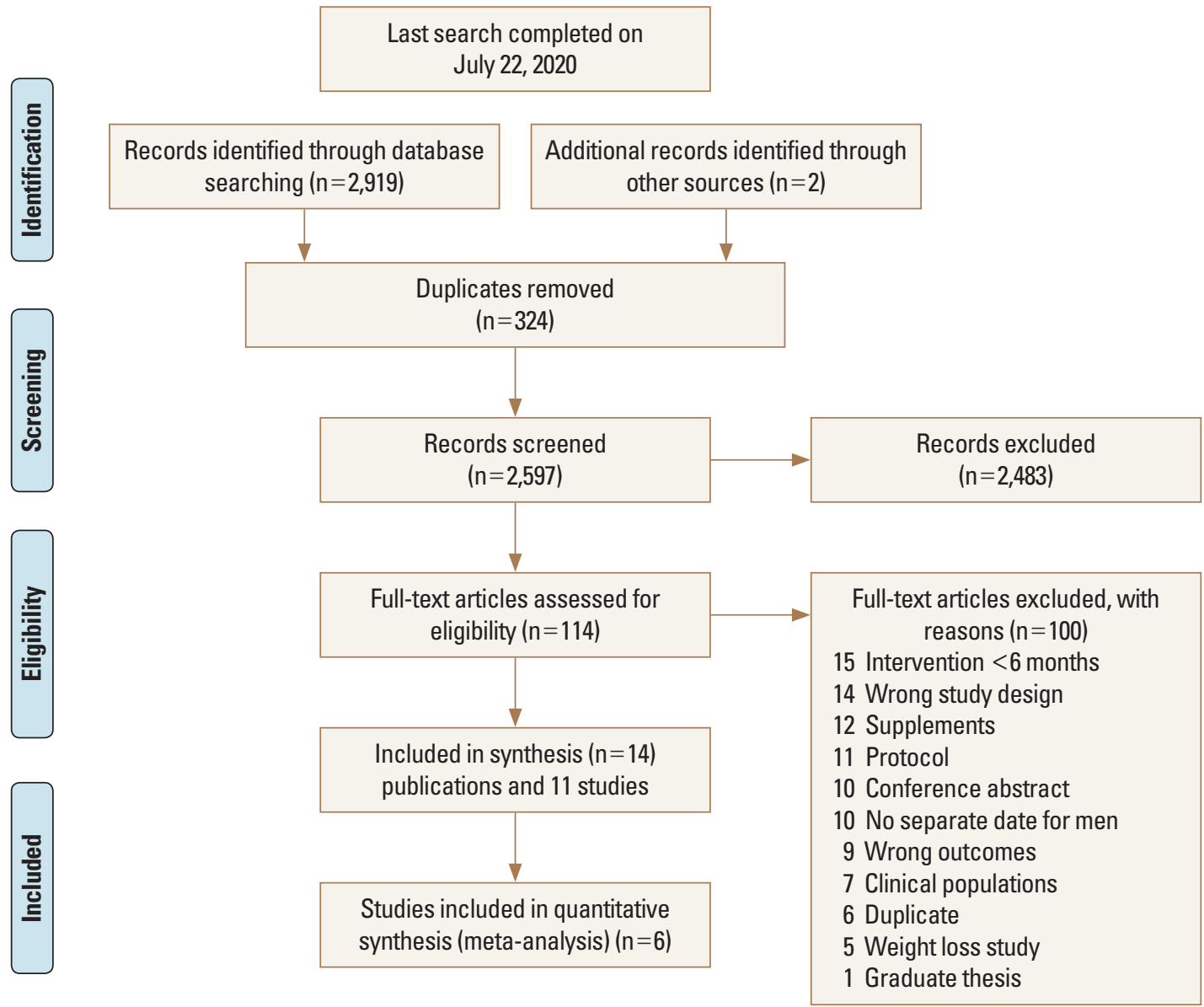

Fig. 1. Preferred Reporting Items for Systematic Reviews and Meta-Analyses flow diagram.

study cohort, but different outcomes.[24-28] In these instances, we collated the data so that each intervention was the unit of interest, rather than each record. We searched PubMed on July 24, 2020 but did not locate errata or retractions for included studies.

\section{Included Studies}

We included 14 publications for 11 studies with $\mathrm{N}=723$ men at final assessment (sample size 17-132 participants), all included studies were RCTs. In 10 of the 11 studies, the mean age for men was $>45$ years of age. Participants were from Asia [29]; Australia [26,27,30,31]; Europe [24,25,32- 


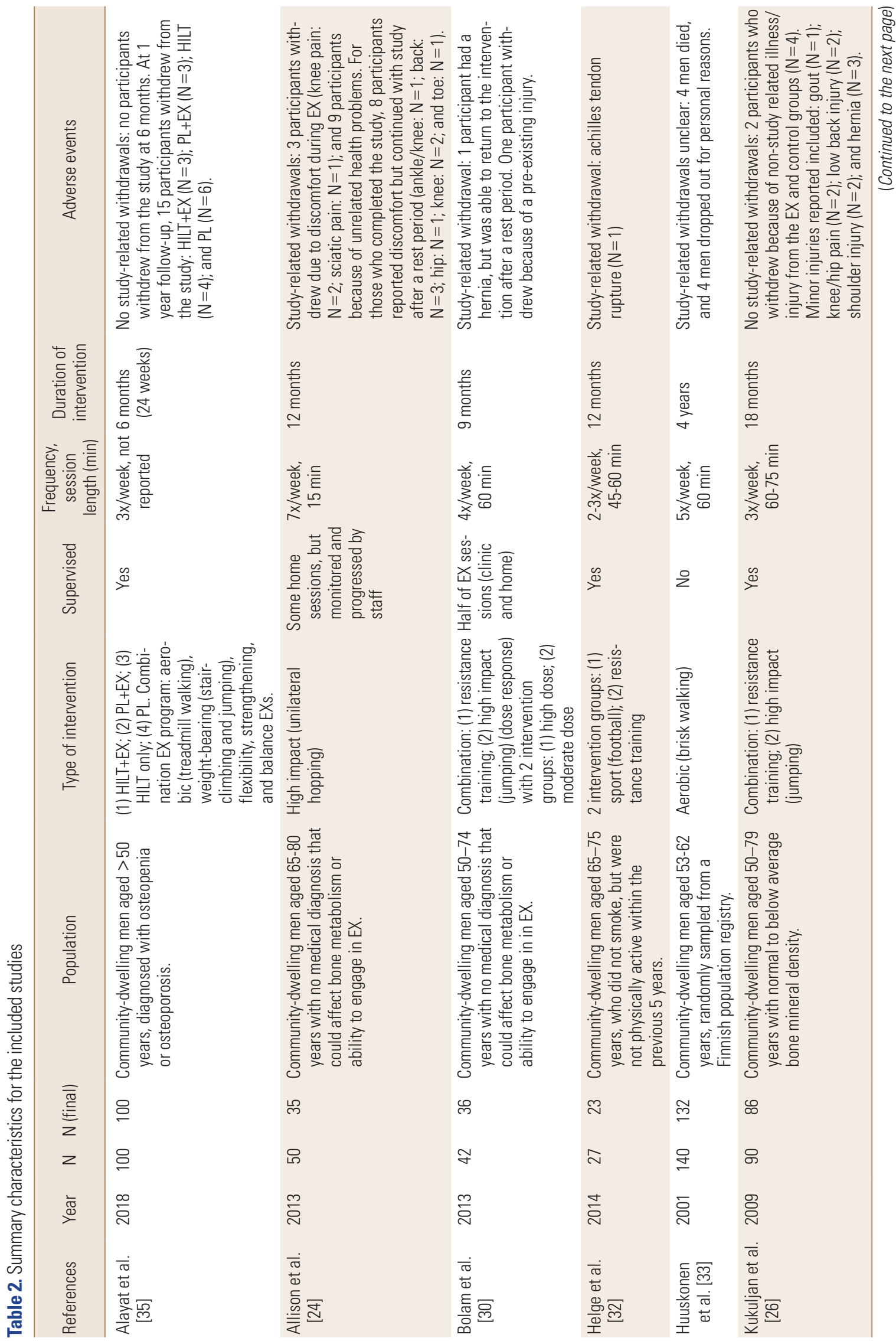




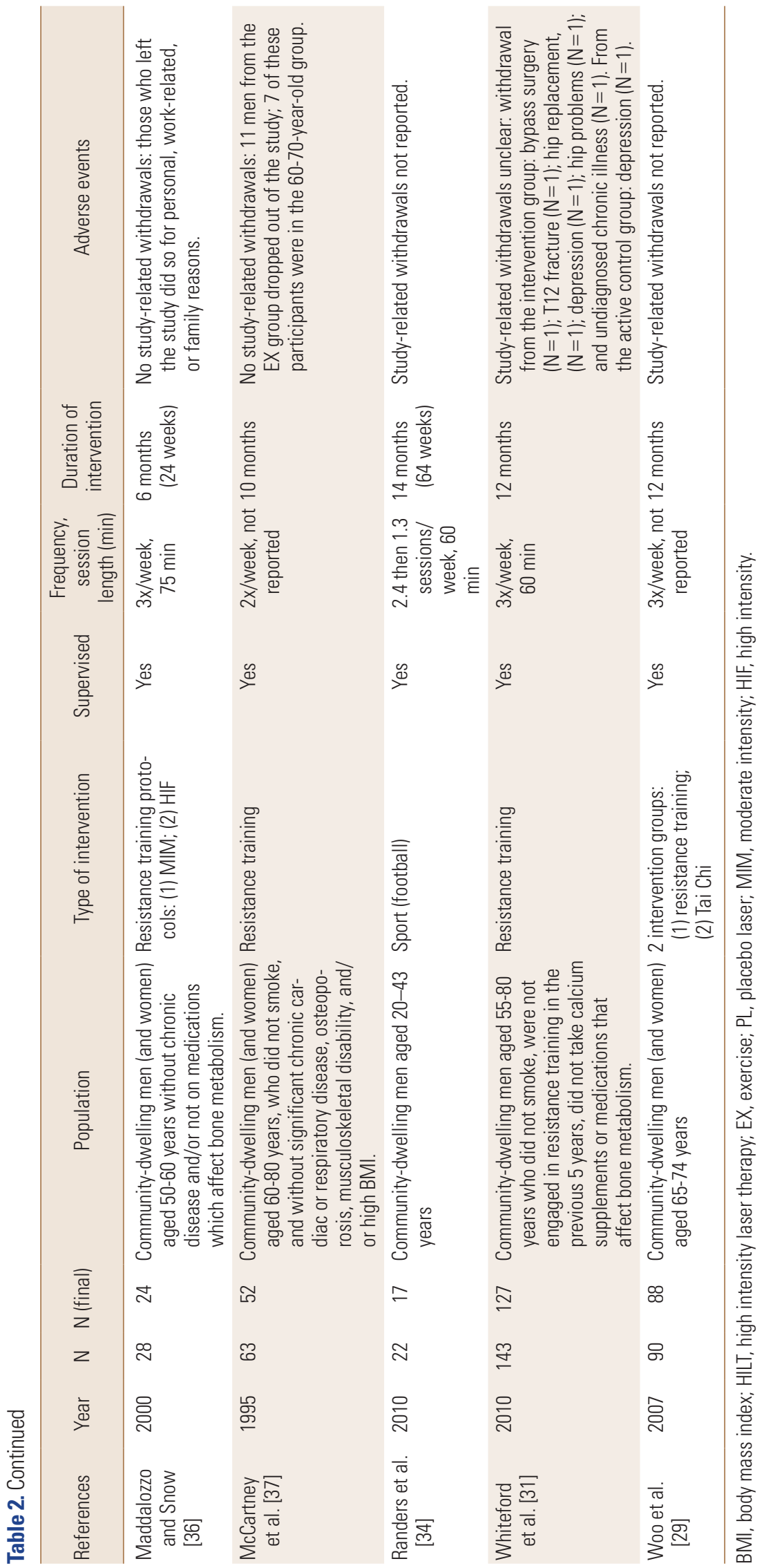


34]; Middle East [35]; and North America [36,37]. Physical activity interventions ranged from 6 months to 4 years and included aerobic, high impact loading, resistance training, sport, Tai Chi, and combined programs. Comparator interventions included usual activity, placebo, and no intervention. There was 1 study that included aerobic training [33]; 3 studies that included high impact, either alone or in combination $[24-27,30] ; 5$ studies included resistance training [29,31,32,36,37]; 2 studies included sport [32,34]; 1 study included Tai Chi [29]; and 3 studies used a combination of physical activity types $[26,27,30,35]$. Two studies compared different types of interventions (Table 2).[29,32]

No included studies had fracture as their primary outcome but fracture was included as an adverse event; 1 study [28] included health-related QOL outcomes and reported no differences after 12 months between exercise (progressive resistance training and impact weight-bearing) and control groups. All included studies assessed bone using dual energy X-ray absorptiometry (DXA; aBMD). Two studies [24-27] reported volumetric BMD (vBMD) and/or bone strength outcomes, but data reported were inconsistent for measurement sites and insufficient data, precluding the inclusion of vBMD in meta-analysis. We included 6 stud- ies in the meta-analysis [29-32,34,35] with $\mathrm{N}=391$ (391/723, $54 \%$ of total sample). Study sample sizes in the meta-analysis ranged from 17 to 127 participants: 3 studies [29,31,35] had a sample size greater than 50 participants (at final assessment). Based on GRADE quality assessments, all outcomes for aBMD, as measured with DXA, were rated as low certainty evidence. Reasons for downgrading the body of evidence for all outcomes was based on (1) many high or unclear risk of bias ratings; and (2) aBMD is a surrogate measure for fracture (indirectness). We did not downgrade for (3) imprecision, however noted that most studies were small; (4) inconsistency because although results varied between studies a possible explanation was the different study populations and physical activity interventions; or (5) publication bias because we conducted a comprehensive search for evidence, and although there were small trials, some studies reported findings with no statistically significant differences.

\section{Risk of Bias}

Please see Figure 2 for a summary of the risk of bias. We were unable to undertake formal statistical tests to investigate funnel plot asymmetry for our primary analysis be-

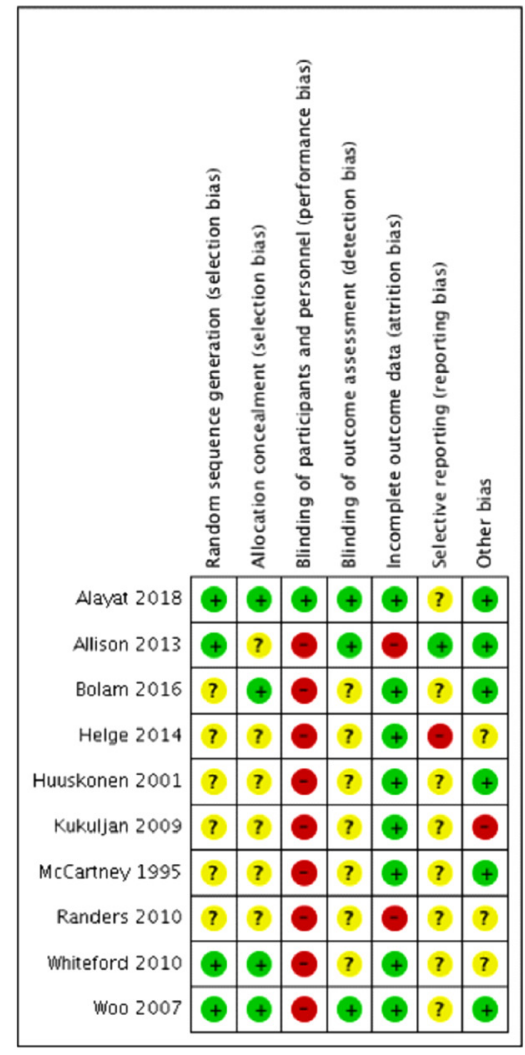

A

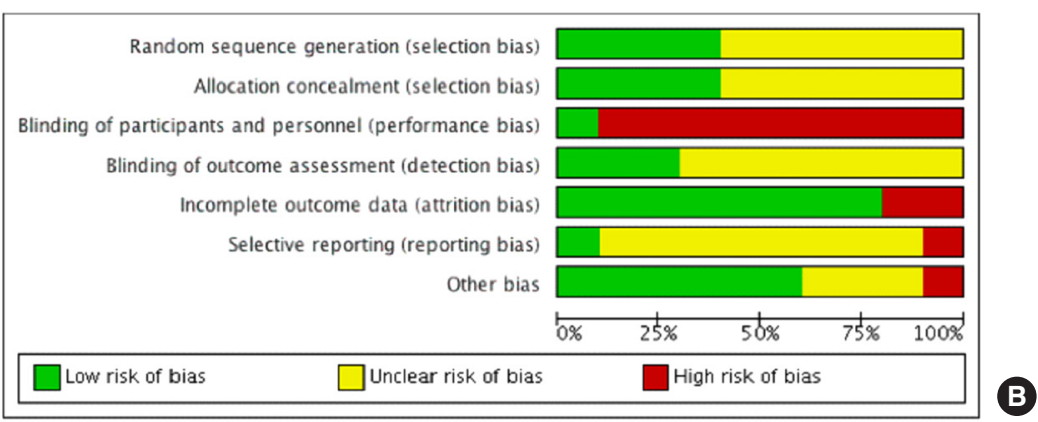

Fig. 2. Risk of bias ratings for individual studies (A) and overall summary of risk of bias for the collective evidence (B). 
cause of the low number of studies ( $<10$ interventions).

\section{Summary of Meta-analysis}

Overall, based on the meta-analysis of aBMD mean difference (MD) and GRADE, there is low certainty evidence that physical activity makes a small difference in aBMD at the total hip (5 RCTs, N=324; MD, 0.03 [95\% Cl, 0.01 to $0.05]$ ); but small to no difference at the femoral neck (3 RCTs, $\mathrm{N}=186 ; \mathrm{MD}, 0.00$ [95\% Cl, -0.04 to 0.04$]$ ); lumbar spine [3 RCTs; $\mathrm{n}=213 ; \mathrm{MD}, 0.05$ [95\% $\mathrm{Cl},-0.01$ to 0.11$]$ ); or whole body (4 RCTs, N=203; MD, $-0.00[95 \% \mathrm{Cl},-0.03$ to $0.02]$ ) aBMD. We did not conduct subgroup analysis due to a lack of available data. Total hip and lumbar spine aBMD results changed using a fixed-effects model. However, we decided to use the fixed-effect model only for the total hip aBMD because of the lower heterogeneity of the pooled effect (47\%) (Fig. 3).

Femoral neck aBMD

$\begin{array}{lll} & \text { Physical Activity } & \text { Control } \\ \text { Study } & \text { Total Mean SD Total Mean SD }\end{array}$

$\begin{array}{lllllll}\text { Bolam 2018 } & 23 & 0.77 & 0.1221 & 13 & 0.78 & 0.1370\end{array}$

$\begin{array}{lllllll}\text { Helge } 2014 & 17 & 0.96 & 0.2011 & 6 & 1.01 & 0.1543\end{array}$

Whiteford 2010

$61 \quad 0.97$

$\begin{array}{lll}66 & 0.97 & 0.1280\end{array}$

Fixed effect model 101

85

Helerogeneity $I^{2}=0 \%, \tau^{2}=0, p=079$

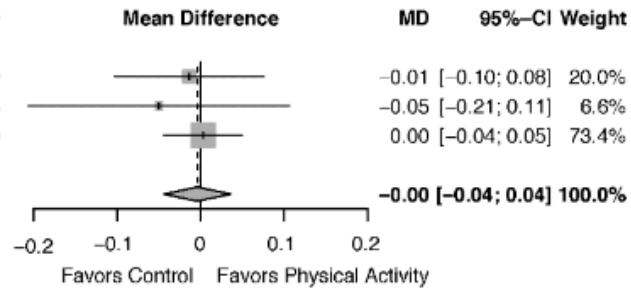

(A)

Total hip aBMD

\begin{tabular}{lrrr} 
Study & \multicolumn{2}{c}{$\begin{array}{c}\text { Physical Activity } \\
\text { Total Mean }\end{array}$} \\
Alayat 2018 & 25 & 0.93 & 0.0400 \\
Bolam 2016 & 23 & 0.95 & 0.1111 \\
Helge 2014 & 17 & 1.02 & 0.1150 \\
Whiteford 2010 & 61 & 1.05 & 0.1570 \\
Woo 2007 & 59 & -0.83 & 2.0364 \\
& & \\
Fixed effect model \\
$\begin{array}{l}\text { Random effects model } \\
\text { Heterogeneity: } t^{2}=47 \%, \tau^{2}=0.0009, p=0.11\end{array}$
\end{tabular}

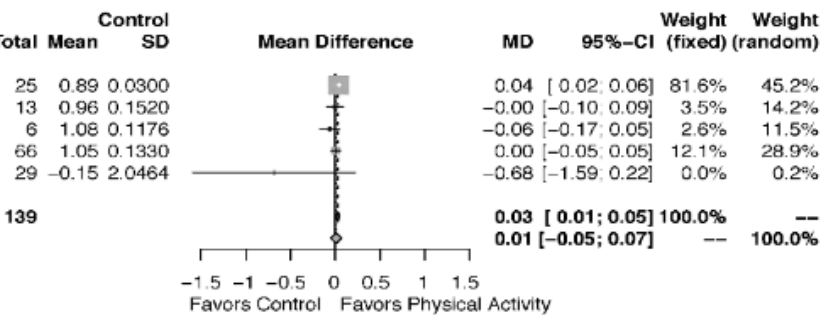

B

Lumbar spine aBMD

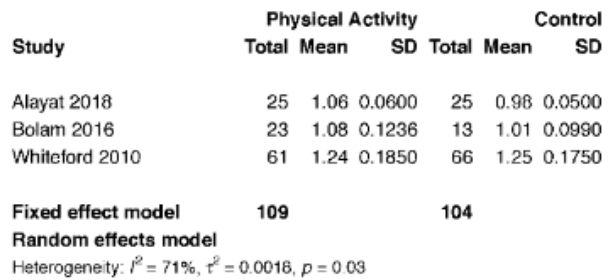

Whole body aBMD

\begin{tabular}{|c|c|c|c|c|c|c|}
\hline \multirow{2}{*}{ Study } & \multicolumn{3}{|c|}{ Physical Activity } & & \multicolumn{2}{|r|}{ Control } \\
\hline & Total & Mean & SD & Total & Mean & SD \\
\hline Bolam 2016 & 23 & 1.19 & 0.0741 & 13 & 1.17 & 0.1070 \\
\hline Helge 2014 & 17 & 1.22 & 0.0915 & 6 & 1.27 & 0.0735 \\
\hline Randers 2010 & 10 & 1.32 & 0.0632 & 7 & 1.28 & 0.0794 \\
\hline Whitelord 2010 & 61 & 1.23 & 0.1080 & 66 & 1.24 & 0.0900 \\
\hline Fixed effect model & 111 & & & 92 & & \\
\hline
\end{tabular}

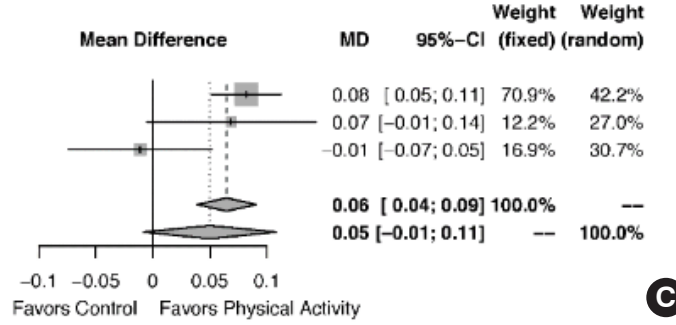

$\odot$

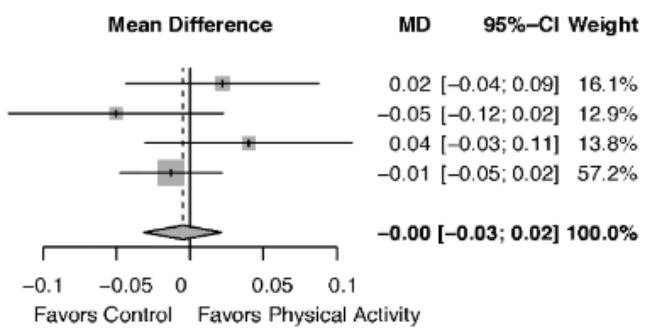

Fig. 3. Forest plots for areal bone mineral density (aBMD) evidence for femoral neck (A), total hip (B), lumbar spine (C), and whole body (D). SD, standard deviation; $\mathrm{MD}$, mean difference; $\mathrm{Cl}$, confidence interval. 


\section{Summary of findings from studies not included in meta-analysis}

Of the 11 studies included in this review, 5 studies [26, $27,33,36,37]$ were not included in the meta-analysis because of insufficient data or different bone outcomes, [24-27, 33] participants acted as their control group, $[24,25,36]$ or bone outcomes not measured by DXA.[36] We note the following findings reported in these publications. In the study by Huuskonen and colleagues [33] there were no differences in BMD between groups or at any skeletal sites after 4 years. In the study by Maddalozzo and Snow [36] where participants' control period aBMD was included as a covariate, there was a significant (group $\times$ time) increase in lumbar spine (1.9\%) and whole-body BMD for high-intensity resistance training. McCartney and colleagues [37] reported "no change in lumbar spine and whole-body BMD as a result of the training program." page B101. There were 2 studies that reported vBMD.[24-27] In the study by Allison and colleagues $[24,25]$, we did not include aBMD outcomes in the meta-analysis because participants' legs were randomized to receive the intervention or controlled conditions (and thus results were not independent). Although the generic inverse variance method could be employed to account for data type, we did not have the standard error of the individual intervention-control differences. Regardless, aBMD was different over time between legs (favoring the intervention) at the femoral neck, but not total hip. For the volumetric bone outcomes, trochanter cortical bone mineral content increased on the exercise leg, and the femoral neck buckling ratio (a measure of "bone instability" defined by the outer bone layer and cortical thickness [38]) decreased more on the exercise leg. There were also significant localized changes at the proximal femur on the leg that completed the exercises. For the study by Kukuljan and colleagues [26,27] and Matthews and colleagues [28] participants in the exercise group increased aBMD at the femoral neck at 12 months (Fig. 2), increased femoral neck cross-sectional area and bone strength (section modulus), and increased lumbar spine aBMD at 12 months (Fig. 2) and trabecular vBMD. No other significant effects for bone outcomes (using DXA or QCT) were noted.

\section{Adverse events}

Across studies, there was inconsistency and lack of clarity in reporting adverse events. Studies did not provide in- formation on if/how adverse or serious adverse events were adjudicated as resulting (or not) from the intervention. For example, the Huuskonen and colleagues [33] reported 3 participants died during the 4 years of the study, but no other information was provided. The number of reported adverse events ranged from 1 [32] to 11 [24,25] (Table 2). Overall, 2 studies did not report on adverse events,[29,34] 2 studies were unclear with reporting events,[31,33] 5 studies reported no adverse events-related withdrawals, [26,27, 30,35-37] and 2 studies reported adverse events-related withdrawals.[24,25,32] From the 2 studies, only one study reported a serious adverse event (Achilles tendon rupture) [32]; while the other study reported musculoskeletal discomfort (knee pain and sciatica).[24,25]

\section{Intervention attendance}

Ten of the 11 included studies reported attendance (number and/or percentage), although in 2 of the studies $[33,35]$ the information provided was unclear, or was not provided separately for men.[37] Attendance for the remaining eight intervention groups ranged from 53\% [30] to 93\% [36]. Specifically, mean attendance was as follows: $90.5 \%[24,25]$; $53 \%$ to $65 \%$ [30]; $66 \%$ to $73 \%$ [32]; $63 \%$ [26,27]; $88 \%$ for men and women [37]; $71 \%$ [31]; $76 \%$ to $81 \%$ [29]; and $93 \%$ [36].

\section{DISCUSSION}

Minimizing fracture risk is important for everyone across the lifespan, however, there are knowledge and practice gaps for physical activity and fracture prevention in men. We cautiously report low certainty evidence for a small effect favoring physical activity for aBMD (a surrogate measure of fracture) at the total hip in men. There are small or no effects for femoral neck, lumbar spine, and whole-body aBMD. We did not locate RCTs of physical activity interventions that reported fracture as a major outcome, importantly from a person-centered perspective only one study reporting QOL, and there were relatively few studies (and with small sample sizes and relatively short duration), and adverse events were inconsistently reported without information for how these events were adjudicated. We further underscore limited evidence (2 included studies) [24-28] that used 3-dimensional (3D) imaging to assess changes in bone outcomes. Given that imaging has advanced in re- 
cent years, it is important for future interventions to use instruments that can provide better characterization of bone structure and strength. Taken together, we report promising (but small) findings for physical activity at the total hip in men; but acknowledge the results should be viewed cautiously, as they might change considerably with new evidence. It is imperative future studies address knowledge gaps to support development of physical activity guidelines specifically for men's bone health.

Our findings extend previous systematic reviews of physical activity and men's bone health [9-13] highlighting gaps in the field: many interventions were shorter in duration, included few participants, and lacked data from 3D bone imaging. We extend evidence from the most recent systematic review from Kemmler and colleagues [13] (last search data November 2016; 8 studies) by incorporating 3 additional studies, including the study with men with osteoporosis, from Alayat and colleagues [35]. We acknowledge that the study by Alayat and colleagues [35] had the highest weighting in the meta-analysis, and the results were not sustained in the sensitivity analysis, however, this study had a lower risk of bias across almost all domains, and the population was older men with a diagnosis of osteoporosis. Further, the exercise intervention included a comprehensive thrice weekly 6 months program of aerobic training (treadmill walking), weight-bearing (including stair-climbing and jumping with supervision), flexibility, strengthening, and balance exercises. Potential corroboration of the results from Alayat and colleagues [35] are observed in the study by Harding and colleagues [39,40]. We were not able to include the study by Harding and colleagues $[39,40]$ in the systematic review because the control group was not based on randomization; however, they also reported a positive effect for aBMD at the hip and lumbar spine for men with low bone mass engaged in a high intensity resistance training and bone loading program.

Exercise intensity may contribute to bone outcomes: higher physical activity intensity or loading may result in greater change in bone outcomes.[41] Although approximately half of the included studies involved higher impact activities (e.g., jumping, football), [24-28,30,32,34,35] we were unable to include 2 of these studies in the meta-analysis.[24-28] Of note, the studies by Allison and colleagues $[24,25]$, Kukuljan and colleagues [26,27], and Matthews and colleagues [28] included higher impact activities and noted improvements in BMD (areal and volumetric) at different regions of the hip, and lumbar spine. Our findings are in line with those reported in a 2011 Cochrane Systematic Review on exercise and bone health in postmenopausal women [7]: small increase in BMD at hip (especially with progressive resistance training) and lumbar spine (combination programs) favoring exercise. However, the body of evidence reporting on the effects of physical activity on bone strength in older women is much larger: our review of studies in men across the lifespan had 11 studies $(\mathrm{N}=723)$ compared with a much larger number of studies in postmenopausal women ( 43 studies; $N=4,320$ ) in the Cochrane review.

We noted several limitations in the identified literature, such as the diversity of the interventions, small sample sizes, (relatively) short duration of interventions, and low quality of evidence. There were also interventions that we could not include, as data from men and women were not reported separately, or they were not provided in the record (and we were unable to make contact with the authors). In addition, during our meta-analysis, we grouped together all types of physical activity and age groups, because of the small number of studies available. Finally, adverse event reporting was not consistent across studies, and this limited our ability to quantitatively evaluate possible harms associated with physical activity. To our knowledge, we are not aware of any other potential biases while conducting this review: we were not authors/investigators on any interventions included in this review; we included publications from all years and all languages; and we conducted a comprehensive search for literature across multiple databases.

In summary, it is important to promote health behaviors to optimize bone health for everyone. Physical activity interventions show promise for men's bone health, but the current evidence must be viewed cautiously. Given the importance of bone health for both men and women, our review highlights a gap in evidence as to the specific intervention prescription that benefits men's bone geometry, structure, microarchitecture and ultimately bone's strength. Future studies should better report possible adverse events, include QOL outcomes, and use more advanced imaging instruments in larger samples (and with a longer timeframe) to support discerning the certainty of the evidence for the effect of different types of physical activity for men's bone health. 


\section{DECLARATIONS}

\section{Acknowledgments}

We gratefully acknowledge the support of Canadian Institutes of Health Research (CIHR), Centre for Hip Health and Mobility, and the Canada Research Chairs program for A/Prof Ashe. Dr. Sale held a CIHR New Investigator Salary Award at the time the review was conducted.

\section{Funding}

This research was supported by a grant from the Canadian Institutes of Health Research.

\section{Authors' contributions}

All authors contributed to the study design, data collection, and/or analysis and interpretation, and approved the final manuscript. IKdS, NYE, and LAB contributed equally to the manuscript.

\section{Ethics approval and consent to participate Not applicable.}

\section{Conflict of interest}

No potential conflict of interest relevant to this article was reported.

\section{ORCID}

Maureen C. Ashe https://orcid.org/0000-0002-6820-4435 Isis Kelly dos Santos https://orcid.org/0000-0001-7615-416X Nicola Y. Edwards https://orcid.org/0000-0002-4224-4666 Joseph H. Puyat https://orcid.org/0000-0003-3710-5773 Lora M Giangregorio https://orcid.org/0000-0002-3739-1805

\section{REFERENCES}

1. Kanis JA, Johnell O, Oden A, et al. Long-term risk of osteoporotic fracture in Malmö. Osteoporos Int 2000;11:669-74. http://dx.doi.org/10.1007/s001980070064.

2. Naves M, Díaz-López JB, Gómez C, et al. Prevalence of osteoporosis in men and determinants of changes in bone mass in a non-selected Spanish population. Osteoporos Int 2005;16:603-9. http://dx.doi.org/10.1007/s00198-0041727-x.

3. Kannegaard PN, van der Mark S, Eiken P, et al. Excess mortality in men compared with women following a hip frac- ture. National analysis of comedications, comorbidity and survival. Age Ageing 2010;39:203-9. http://dx.doi.org/10. 1093/ageing/afp221.

4. Binkley N. A perspective on male osteoporosis. Best Pract Res Clin Rheumatol 2009;23:755-68. http://dx.doi.org/10. 1016/j.berh.2009.10.001.

5. Feldstein AC, Nichols G, Orwoll E, et al. The near absence of osteoporosis treatment in older men with fractures. Osteoporos Int 2005;16:953-62. http://dx.doi.org/10.1007/ s00198-005-1950-0.

6. Tarride JE, Guo N, Hopkins R, et al. The burden of illness of osteoporosis in Canadian men. J Bone Miner Res 2012;27: 1830-8. http://dx.doi.org/10.1002/jbmr.1615.

7. Howe TE, Shea B, Dawson LJ, et al. Exercise for preventing and treating osteoporosis in postmenopausal women. Cochrane Database Syst Rev 2011:CD000333. http://dx.doi. org/10.1002/14651858.CD000333.pub2.

8. Sherrington C, Fairhall N, Wallbank G, et al. Exercise for preventing falls in older people living in the community: an abridged Cochrane systematic review. Br J Sports Med 2020;54:885-91. http://dx.doi.org/10.1136/bjsports-2019101512.

9. Bolam KA, van Uffelen JG, Taaffe DR. The effect of physical exercise on bone density in middle-aged and older men: a systematic review. Osteoporos Int 2013;24:2749-62. http: //dx.doi.org/10.1007/s00198-013-2346-1.

10. Gómez-Cabello A, Ara I, González-Agüero A, et al. Effects of training on bone mass in older adults: a systematic review. Sports Med 2012;42:301-25. http://dx.doi.org/10.2165/ 11597670-000000000-00000.

11. Kelley GA, Kelley KS, Kohrt WM. Exercise and bone mineral density in men: a meta-analysis of randomized controlled trials. Bone 2013;53:103-11. http://dx.doi.org/10.1016/ j.bone.2012.11.031

12. Kelley GA, Kelley KS, Tran ZV. Exercise and bone mineral density in men: a meta-analysis. J Appl Physiol (1985) 2000; 88:1730-6. http://dx.doi.org/10.1152/jappl.2000.88.5.1730.

13. Kemmler W, Shojaa M, Kohl M, et al. Exercise effects on bone mineral density in older men: a systematic review with special emphasis on study interventions. Osteoporos Int 2018;29:1493-504. http://dx.doi.org/10.1007/s00198018-4482-0.

14. Liberati A, Altman DG, Tetzlaff J, et al. The PRISMA statement for reporting systematic reviews and meta-analyses of studies that evaluate healthcare interventions: expla- 
nation and elaboration. BMJ 2009;339:b2700. http://dx. doi.org/10.1136/bmj.b2700.

15. Guyatt GH, Oxman AD, Vist GE, et al. GRADE: an emerging consensus on rating quality of evidence and strength of recommendations. BMJ 2008;336:924-6. http://dx.doi.org/ 10.1136/bmj.39489.470347.AD.

16. Lefebvre C, Manheimer E, Glanville J. Chapter 6. Searching for studies. In: Higgins JPT, Green S, editors. Cochrane handbook for systematic reviews of interventions: Version 5.1.0. Chichester, UK: The Cochrane Collaboration; 2011.

17. Stewart F, Fraser C, Robertson C, et al. Are men difficult to find? Identifying male-specific studies in MEDLINE and Embase. Syst Rev 2014;3:78. http://dx.doi.org/10.1186/ 2046-4053-3-78.

18. Higgins JP, Altman DG, Gøtzsche PC, et al. The Cochrane Collaboration's tool for assessing risk of bias in randomised trials. BMJ 2011;343:d5928. http://dx.doi.org/10.1136/ bmj.d5928.

19. GRADEpro GDT. GRADEpro guideline development tool [Software]. Hamilton, ON: McMaster University; 2015.

20. Higgins JPT, J DJ. Chapter 7. Selecting studies and collecting data. In: Higgins JPT, Green S, editors. Cochrane handbook for systematic reviews of interventions: Version 5.1.0. Chichester, UK: The Cochrane Collaboration; 2011.

21. Cornell JE, Mulrow CD, Localio R, et al. Random-effects meta-analysis of inconsistent effects: a time for change. Ann Intern Med 2014;160:267-70. http://dx.doi.org/10.7326/ m13-2886.

22. The R Development Core Team. R: A language and environment for statistical computing. Version 4.0.3. Vienna, AT: R Foundation for Statistical Computing; 2020.

23. Balduzzi S, Rücker G, Schwarzer G. How to perform a meta-analysis with R: a practical tutorial. Evid Based Ment Health 2019;22:153-60. http://dx.doi.org/10.1136/ebmental-2019-300117.

24. Allison SJ, Folland JP, Rennie WJ, et al. High impact exercise increased femoral neck bone mineral density in older men: a randomised unilateral intervention. Bone 2013; 53:321-8. http://dx.doi.org/10.1016/j.bone.2012.12.045.

25. Allison SJ, Poole KE, Treece GM, et al. The influence of highimpact exercise on cortical and trabecular bone mineral content and 3D distribution across the proximal femur in older men: A randomized controlled unilateral intervention. J Bone Miner Res 2015;30:1709-16. http://dx.doi. org/10.1002/jbmr.2499.
26. Kukuljan S, Nowson CA, Bass SL, et al. Effects of a multicomponent exercise program and calcium-vitamin-D3fortified milk on bone mineral density in older men: a randomised controlled trial. Osteoporos Int 2009;20:1241-51. http://dx.doi.org/10.1007/s00198-008-0776-y.

27. Kukuljan S, Nowson CA, Sanders KM, et al. Independent and combined effects of calcium-vitamin D3 and exercise on bone structure and strength in older men: an 18-month factorial design randomized controlled trial. J Clin Endocrinol Metab 2011;96:955-63. http://dx.doi.org/10.1210/ jc.2010-2284.

28. Matthews J, Torres SJ, Milte CM, et al. Effects of a multicomponent exercise program combined with calcium-vitamin $D(3)$-enriched milk on health-related quality of life and depressive symptoms in older men: secondary analysis of a randomized controlled trial. Eur J Nutr 2020;59: 1081-91. http://dx.doi.org/10.1007/s00394-019-01969-8.

29. Woo J, Hong A, Lau E, et al. A randomised controlled trial of Tai Chi and resistance exercise on bone health, muscle strength and balance in community-living elderly people. Age Ageing 2007;36:262-8. http://dx.doi.org/10.1093/ ageing/afm005.

30. Bolam K, Skinner T, Taaffe DR. The effect of high-impact exercise on hip and spine bone density in communitydwelling middle-aged and older men: A 9-month doseresponse randomised controlled trial. J Sci Med Sport 2013; 16:E14-5. http://dx.doi.org/10.1016/j.jsams.2013.10.036.

31. Whiteford J, Ackland TR, Dhaliwal SS, et al. Effects of a 1-year randomized controlled trial of resistance training on lower limb bone and muscle structure and function in older men. Osteoporos Int 2010;21:1529-36. http://dx.doi.org/10.1007/ s00198-009-1132-6.

32. Helge EW, Andersen TR, Schmidt JF, et al. Recreational football improves bone mineral density and bone turnover marker profile in elderly men. Scand J Med Sci Sports 2014; 24 Suppl 1:98-104. http://dx.doi.org/10.1111/sms.12239.

33. Huuskonen J, Väisänen SB, Kröger $H$, et al. Regular physical exercise and bone mineral density: a four-year controlled randomized trial in middle-aged men. The DNASCO study. Osteoporos Int 2001;12:349-55. http://dx.doi. org/10.1007/s001980170101.

34. Randers MB, Nielsen JJ, Krustrup BR, et al. Positive performance and health effects of a football training program over 12 weeks can be maintained over a 1-year period with reduced training frequency. Scand J Med Sci Sports 
2010;20 Suppl 1:80-9. http://dx.doi.org/10.1111/j.16000838.2010.01091.x.

35. Alayat MSM, Abdel-Kafy EM, Thabet AAM, et al. Long-term effect of pulsed Nd-YAG laser combined with exercise on bone mineral density in men with osteopenia or osteoporosis: 1 year of follow-up. Photomed Laser Surg 2018;36: 105-11. http://dx.doi.org/10.1089/pho.2017.4328.

36. Maddalozzo GF, Snow CM. High intensity resistance training: effects on bone in older men and women. Calcif Tissue Int 2000;66:399-404. http://dx.doi.org/10.1007/s002 230010081.

37. McCartney N, Hicks AL, Martin J, et al. Long-term resistance training in the elderly: effects on dynamic strength, exercise capacity, muscle, and bone. J Gerontol A Biol Sci Med Sci 1995;50:B97-104. http://dx.doi.org/10.1093/gero na/50a.2.b97.

38. Anitha $D$, Lee T. Assessing bone quality in terms of bone mineral density, buckling ratio and critical fracture load. J
Bone Metab 2014;21:243-7. http://dx.doi.org/10.11005/ jbm.2014.21.4.243.

39. Harding AT, Weeks BK, Lambert C, et al. Effects of supervised high-intensity resistance and impact training or machine-based isometric training on regional bone geometry and strength in middle-aged and older men with low bone mass: The LIFTMOR-M semi-randomised controlled trial. Bone 2020;136:115362. http://dx.doi.org/10.1016/ j.bone.2020.115362.

40. Harding AT, Weeks BK, Lambert C, et al. A comparison of bone-targeted exercise strategies to reduce fracture risk in middle-aged and older men with osteopenia and osteoporosis: LIFTMOR-M semi-randomized controlled trial. J Bone Miner Res 2020;35:1404-14. http://dx.doi.org/10. 1002/jbmr.4008.

41. Hong AR, Kim SW. Effects of resistance exercise on bone health. Endocrinol Metab (Seoul) 2018;33:435-44. http:// dx.doi.org/10.3803/EnM.2018.33.4.435. 
Supplementary Appendix 1. MEDLINE search strategy

\begin{tabular}{|c|c|}
\hline No. & Medical Subject Heading (MeSH) or keywords \\
\hline 1 & osteoporosis/ \\
\hline 2 & osteoporo\$.tw. \\
\hline 3 & osteopenia.tw. \\
\hline 4 & bone loss\$.tw. \\
\hline 5 & bone mineral densit\$.tw. \\
\hline 6 & decalcifi\$.tw. \\
\hline 7 & deminerali?ed bone.tw. \\
\hline 8 & or/1-7 \\
\hline 9 & exp exercise/ \\
\hline 10 & exp exercise therapy/ \\
\hline 11 & physical fitness/ \\
\hline 12 & physical activit\$.tw. \\
\hline 13 & vibration/tu \\
\hline 14 & vibration therap\$.mp. \\
\hline 15 & Physical Therapy Modalities/ \\
\hline 16 & Physical Exertion/ \\
\hline 17 & exp Physical Endurance/ \\
\hline 18 & exp Sports/ \\
\hline 19 & Pliability/ \\
\hline 20 & exertion\$.tw. \\
\hline 21 & exercis\$.tw. \\
\hline 22 & sport\$.tw. \\
\hline 23 & ((physical or motion) adj5 (fitness or therapy or therapies)).tw \\
\hline 24 & (physical\$ adj2 endur\$).tw. \\
\hline 25 & weight train\$.tw. \\
\hline 26 & or/9-25 \\
\hline 27 & 8 and 26 \\
\hline 28 & 27 and (male or males or men).tw. \\
\hline 29 & 27 and Male/ \\
\hline 30 & 28 or 29 \\
\hline 31 & 26 and 30 \\
\hline 32 & randomized controlled trial.pt. \\
\hline 33 & controlled clinical trial.pt. \\
\hline 34 & randomized.ab. \\
\hline 35 & placebo.ab. \\
\hline 36 & clinical trials as topic.sh. \\
\hline 37 & randomly.ab. \\
\hline 38 & trial.ti. \\
\hline 39 & or/32-38 \\
\hline 40 & exp animals/ not humans.sh. \\
\hline 41 & 39 not 40 \\
\hline 42 & 31 and 41 \\
\hline
\end{tabular}

